\title{
Self-Organization of Atmospheric Macroturbulence into Critical States of Weak Nonlinear Eddy-Eddy Interactions
}

\author{
TAPio Schneider And Christopher C. WALKer \\ California Institute of Technology, Pasadena, California
}

(Manuscript received 7 June 2005, in final form 1 November 2005)

\begin{abstract}
It is generally held that atmospheric macroturbulence can be strongly nonlinear. Yet weakly nonlinear models successfully account for scales and structures of baroclinic eddies in Earth's atmosphere. Here a theory and simulations with an idealized GCM are presented that suggest weakly nonlinear models are so successful because atmospheric macroturbulence organizes itself into critical states of weak nonlinear eddy-eddy interactions. By modifying the thermal structure of the extratropical atmosphere such that its supercriticality remains limited, macroturbulence inhibits nonlinear eddy-eddy interactions and the concomitant inverse energy cascade from the length scales of baroclinic instability to larger scales. For small meridional surface temperature gradients, the extratropical thermal stratification and tropopause height are set by radiation and convection, and the supercriticality is less than one; for sufficiently large meridional surface temperature gradients, the extratropical thermal stratification and tropopause height are modified by baroclinic eddies such that the supercriticality does not significantly exceed one. In either case, the scale of the energy-containing eddies is similar to the scale of the linearly most unstable baroclinic waves, and eddy kinetic and available potential energies are equipartitioned. The theory and simulations point to fundamental constraints on the thermal structures and global circulations of the atmospheres of Earth and other planets, for example, by providing limits on the tropopause height and estimates for eddy scales, eddy energies, and jet separation scales.
\end{abstract}

\section{Introduction}

In the extratropics of Earth's atmosphere, the length scales $(\sim 1000 \mathrm{~km})$, time scales $(\sim 2$ days $)$, and structures of the energy-containing eddies resemble those of the linearly most unstable baroclinic waves (e.g., Simmons and Hoskins 1976, 1977; Lau 1978; Frederiksen 1983; Valdes and Hoskins 1988). Nonetheless, the view prevails that eddy energy can cascade from the length scales of baroclinic instability to larger scales, implying that the resemblance of the energy-containing eddies and the linearly most unstable waves in Earth's atmosphere is a coincidence and not a fundamental property of atmospheric macroturbulence. It is thought that from the length scales of baroclinic instability, where potential energy generated by the differential heating of the atmosphere is converted into eddy kinetic energy, the barotropic component of eddy kinetic energy

Corresponding author address: Tapio Schneider, California Institute of Technology, Mail Code 100-23, 1200 E. California Blvd., Pasadena, CA 91125.

E-mail: tapio@caltech.edu cascades to larger scales, as in two-dimensional turbulence, in an inverse energy cascade mediated by nonlinear eddy-eddy interactions (Charney 1971; Salmon 1980). Unless dissipation or the limited size of the planet arrest the inverse cascade at a smaller scale, the energy cascades up to and accumulates near the Rhines scale, where it is channeled into zonal jets and Rossby waves (Rhines 1975; Vallis and Maltrud 1993). Such inverse energy cascades from the scales of the linearly most unstable baroclinic waves to larger Rhines scales have been simulated with two-dimensional and quasigeostrophic models (e.g., Panetta 1993; Cho and Polvani 1996; Held and Larichev 1996; Lapeyre and Held 2003).

In Earth's atmosphere, however, there is no evidence for an inverse energy cascade beyond the scale of the linearly most unstable baroclinic waves. The barotropic eddy kinetic energy spectrum of Earth's atmosphere, shown in Fig. 1, does not exhibit the $-5 / 3$ power-law range at large scales that would be expected in an inverse energy cascade. The energy-containing scale (approximately the scale at which the barotropic eddy kinetic energy is maximal, details will be discussed below), the Rossby radius, and the Rhines scale are all of 


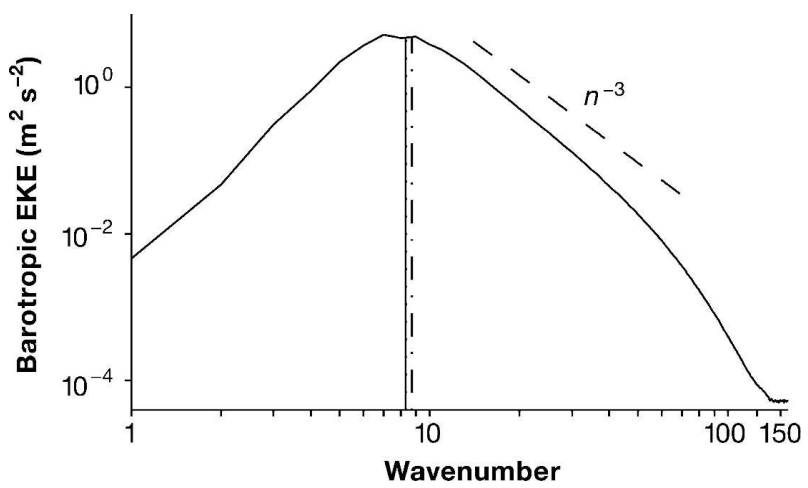

FIG. 1. Annual mean spectrum of barotropic eddy kinetic energy (EKE) according to ECMWF reanalysis data for the years 1980-2001 (Kållberg et al. 2004). The horizontal axis is the spherical wavenumber $n$. The dashed line indicates an $n^{-3}$ power law. The barotropic eddy kinetic energy spectrum is based on the spectrum of the barotropic (vertically averaged) velocities, without the zonal-wavenumber zero component. Vertical lines indicate characteristic length scales expressed as spherical wavenumbers, computed and with $O(1)$ constants as in section 3 and appendix D: energy-containing wavenumber (solid), Rhines wavenumber (dotted), and Rossby wavenumber (dash-dotted), listed in order of increasing wavenumber (energy-containing wavenumber and Rhines wavenumber are almost indistinguishable).

the same order of magnitude. Consistent with the barotropic eddy kinetic energy spectrum, transient eddy spectra indicate upscale kinetic energy transfer from moderately smaller scales up to the scale of the linearly most unstable baroclinic waves, but not or not significantly beyond it (Boer and Shepherd 1983; Shepherd 1987; Straus and Ditlevsen 1999). ${ }^{1}$

In quasigeostrophic models, on which the current understanding of atmospheric macroturbulence is based, the thermal stratification of the atmosphere is taken to be fixed. However, baroclinic eddies can stabilize the extratropical thermal stratification, which opens up the possibility of eddy-mean flow interactions that are not taken into account in quasigeostrophic models and that may inhibit nonlinear eddy-eddy interactions and the inverse energy cascade. Such eddy-mean flow interactions, which may in themselves represent an upscale

\footnotetext{
${ }^{1}$ The higher energies at large scales seen in the spectra shown by Straus and Ditlevsen (1999) are due to variations of the zonal mean flow, which the temporal filtering of Straus and Ditlevsen, unlike the filtering of the zonal-wavenumber zero component in Fig. 1, does not remove completely. Also, Straus and Ditlevsen, as well as Boer and Shepherd (1983) and Shepherd (1987), show spectra of total eddy kinetic energy (vertically averaged eddy kinetic energy), rather than the spectrum of barotropic eddy kinetic energy (eddy kinetic energy of vertically averaged flow) shown in Fig. 1; however, the shapes of the spectra of total and barotropic eddy kinetic energy are similar.
}

energy transfer, albeit not one associated with a cascade of eddy energy, are the focus of the present paper.

We estimate, in terms of the near-surface thermal structure, the pressure range over which baroclinic eddies stabilize the extratropical thermal stratification, define a supercriticality as a ratio of this pressure range estimate to the pressure difference between surface and tropopause, and demonstrate that the so-defined supercriticality does not significantly exceed one in simulations of a wide range of circulations with an idealized GCM (section 2). That the supercriticality does not significantly exceed one implies that the scale of the energy-containing eddies is similar to the scale of the linearly most unstable baroclinic waves, that there is no inverse energy cascade beyond that scale, and that nonlinear eddy-eddy interactions are weak (section 3). From the weakness of nonlinear eddy-eddy interactions, it follows that eddy available potential energy and barotropic and baroclinic eddy kinetic energies are equipartitioned, as they are in the linearly most unstable baroclinic waves (section 4). We discuss to what extent these results constrain the thermal structures and global circulations of the atmospheres of Earth and other planets and to what extent they may have to be modified to take latent heat release in phase changes of water into account (section 5). The appendices contain details of theoretical developments, a description of the idealized GCM, and details on the estimation of flow statistics and the definitions of eddy scales.

\section{Vertical extent of baroclinic entropy fluxes and supercriticality}

\section{a. Theory}

An estimate of the altitude or pressure range over which baroclinic eddies stabilize the thermal stratification by redistributing entropy from the surface into the interior atmosphere can be derived from the zonal momentum balance. Zonal momentum balance, in the temporal and zonal mean at each latitude, requires that the meridional eddy fluxes of surface potential temperature $\theta_{s}$ and of potential vorticity $P$ along isentropes satisfy the balance condition ${ }^{2}$ (Schneider 2004, 2005)

\footnotetext{
${ }^{2}$ Overbars denote temporal and zonal means: $\overline{(\cdot)}^{s}$ along the surface; $\overline{(\cdot)}$ along isentropes if the argument depends on a vertical coordinate; and $\overline{(\cdot)} *{\overline{\left(\rho_{\theta} \cdot\right)}}^{*} \bar{\rho}_{\theta}$ along isentropes weighted by the isentropic density $\rho_{\theta}=-g^{-1} \partial_{\theta} p \mathcal{H}\left(\theta-\theta_{s}\right)$, where $\mathcal{H}(\cdot)$ is the Heaviside step function. Primes denote fluctuations about the respective means. The subscript $s$ marks surface quantities; $\theta$ is the potential temperature; $\bar{\rho}_{\theta}^{0}$ is the mean isentropic density at the mean surface potential temperature $\bar{\theta}_{s} ; v$ is the meridional velocity and $\tilde{v}$ its geostrophic component.
} 


$$
\int_{\theta_{b}}^{\theta_{e}} \frac{\bar{\rho}_{\theta}{\overline{v^{\prime} P^{\prime}}}^{*}}{\bar{P}^{*}} d \theta \approx-\bar{\rho}_{\theta}^{0}{\overline{\tilde{v}_{s}^{\prime} \theta_{s}^{\prime}}}^{s} .
$$

The balance condition states that the isentropic mass fluxes associated with the eddy flux of potential vorticity along isentropes (left-hand side) and with the eddy flux of surface potential temperature (right-hand side) balance each other. The potential temperature $\theta_{b}(\phi)$ is the lowest surface potential temperature that occurs at the latitude $\phi$ under consideration; the potential temperature $\theta_{e}(\phi)$ is the potential temperature up to which baroclinic eddies effect significant mean entropy fluxes (or mean mass fluxes along isentropes). With diffusive eddy flux closures $\overline{v^{\prime} P^{\prime}} * \sim-\left.D \partial_{y} \bar{P}^{*}\right|_{\theta}$ and ${\overline{\tilde{v}_{s}^{\prime} \theta_{s}^{\prime}}}^{s} \sim$ $-D \partial_{y} \bar{\theta}_{s}$ in which the eddy diffusivity $D$ may vary with latitude but is taken to have no essential vertical structure below the upper bound $\theta_{e}$ of the integration, the balance condition (1) leads to the estimate

$$
p_{s}-p_{e} \sim \frac{f}{\beta} \frac{\partial_{y} \bar{\theta}_{s}}{2 \overline{\partial_{p} \theta} s},
$$

where $f=2 \Omega \sin (\phi)$ is the Coriolis parameter and $\beta=$ $d f / d y$ is its meridional derivative (see appendix A for a derivation and a comparison with previous estimates, such as those based on quasigeostrophic baroclinicadjustment hypotheses). The difference between the mean surface pressure $\bar{p}_{s}$ and the mean pressure $\bar{p}_{e}=$ $\bar{p}\left(\theta_{e}\right)$ up to which baroclinic eddies redistribute entropy depends on the meridional potential temperature gradient $\partial_{y} \bar{\theta}_{s}$ and on the static stability $-\bar{\partial}_{p} \theta$ at the surface (or immediately above a near-surface mixed layer).

The adequacy of the estimate (2) depends on the validity of the assumption that the eddy diffusivity has no essential vertical structure, implying vertically uniform mixing, in the pressure range over which baroclinic eddies redistribute entropy. [Eddy diffusivities may be poorly defined or large in or near regions where the potential vorticity gradient vanishes, but as long as the potential vorticity fluxes in those regions vanish or are small, the structure of the eddy diffusivity in those regions is not essential for the adequacy of the estimate (2); see Schneider (2004) for examples of diffusivities from GCM simulations.] We examine this assumption in the limiting cases of strongly and weakly nonlinear macroturbulence, that is, in the limiting cases of strong and weak nonlinear eddy-eddy interactions.

In strongly nonlinear macroturbulence, the inverse energy cascade to large horizontal and vertical scales would lead to barotropization of the energy-containing eddies (Smith and Vallis 2002). Therefore, since the energy-containing eddies dominate the advection of potential vorticity and surface potential temperature fluc- tuations (Held and Larichev 1996), the assumption of vertically uniform mixing is justifiable. Further, we expect that in strongly macroturbulent regions, the energy-containing eddies, potentially with convection coupled to them, dominate the entropy redistribution between surface and tropopause. This implies that the pressure range over which baroclinic eddies redistribute entropy defines the troposphere and that the estimate (2) holds with the mean tropopause pressure $\bar{p}_{t}$ in place of $\bar{p}_{e}$ (Held 1982; Schneider 2004). Defining the bulk stability ${ }^{3}$

$$
\Delta_{v}=-2{\overline{\partial_{p} \theta}}^{s}\left(\bar{p}_{s}-\bar{p}_{t}\right)
$$

and the supercriticality

$$
S_{c}=-\frac{f}{\beta} \frac{\partial_{y} \bar{\theta}_{s}}{\Delta_{v}},
$$

a nondimensional measure of the slope of near-surface isentropes or a ratio of pressure ranges $S_{c} \sim$ $\left(\bar{p}_{s}-\bar{p}_{e}\right) /\left(\bar{p}_{s}-\bar{p}_{t}\right)$, we conclude that macroturbulence would modify the surface potential temperature gradient and the thermal stratification such that $S_{c} \sim 1$. In quasigeostrophic models, however, counterparts of the supercriticality $S_{c}$ are measures of instability: the limit of strongly unstable flow and strongly nonlinear eddyeddy interactions corresponds to $S_{c} \gg 1$ (Pedlosky 1970, 1979; Held and Larichev 1996). The result that strongly nonlinear macroturbulence would modify the surface potential temperature gradient and the thermal stratification such that the supercriticality is close to the critical value $S_{c} \sim 1$ suggests that the modification of the thermal structure inhibits nonlinear eddy-eddy interactions and renders the strongly nonlinear limit unattainable.

In weakly nonlinear macroturbulence, mean flow properties such as the thermal stratification determine the vertical structure of baroclinic eddies. Nonetheless, the streamfunctions of the most unstable linear or weakly nonlinear waves vary only weakly in the pressure range over which baroclinic eddies redistribute entropy in Earth's atmosphere (Simmons and Hoskins 1976, 1977; Valdes and Hoskins 1988). To the extent that the vertical structure of the eddy diffusivity is similar to that of the streamfunction of the most unstable wave-the streamfunction scales with the product of an eddy length scale and an eddy velocity scale and so scales like an eddy diffusivity (Holloway 1986; Kushner

\footnotetext{
${ }^{3}$ Appendix A shows that for Earth-like atmospheres, in which the tropopause height is somewhat greater than the scale height, the bulk stability (3) is approximately equal to the potential temperature difference between tropopause and surface, $\Delta_{v} \sim \bar{\theta}_{t}-\bar{\theta}_{s}$.
} 
and Held 1998) - the assumption of vertically uniform mixing thus remains plausible for rough estimates for Earth's and similar atmospheres (cf. Held 1978). For such atmospheres, we expect the estimate (2) to continue to hold. In regions in which baroclinic eddies, again potentially with convection coupled to them, are sufficiently energetic to dominate the entropy redistribution and modify the thermal stratification between surface and tropopause, we expect the estimate (2) to hold with the mean tropopause pressure $\bar{p}_{t}$ in place of $\bar{p}_{e}$. In regions in which baroclinic eddies are not sufficiently energetic to dominate the entropy redistribution between surface and tropopause and the tropopause and thermal stratification at least in the upper troposphere are set by other processes (e.g., radiation and convection), we expect the estimate (2) for $\bar{p}_{e}$ to be greater than or equal to the mean tropopause pressure $\bar{p}_{t}$. That is, baroclinic entropy fluxes may extend up to the tropopause, or they may be shallower. For the supercriticality, we conclude $S_{c} \lesssim 1$ with $S_{c} \sim 1$ in regions in which baroclinic eddies dominate the entropy redistribution between surface and tropopause and baroclinic entropy fluxes extend to the tropopause. This is in accord with quasigeostrophic models, in which the linear or weakly nonlinear limit corresponds to $S_{c} \lesssim 1$.

\section{b. Simulations}

With an idealized GCM, we investigated whether the thermal structure of an atmosphere indeed adjusts such that $S_{c} \lesssim 1$. The GCM resolves large-scale baroclinic eddies but is idealized in that the surface of the simulated planets has no topography, and radiative heating and cooling are represented by Newtonian relaxation of temperatures toward statically unstable radiativeequilibrium states (see appendix B for a model description). Phase changes of water vapor are not explicitly taken into account. However, if a layer is statically unstable relative to a specified convective temperature lapse rate, a convection scheme mimics latent heat release by relaxing temperatures in that layer toward an enthalpy-conserving profile with the convective lapse rate. The convective lapse rate is $\gamma \Gamma_{d}$, where $\Gamma_{d}=g / c_{p}$ $=9.8 \mathrm{~K} \mathrm{~km}^{-1}$ is the dry adiabatic lapse rate and $\gamma \leq 1$ is a rescaling parameter. For $\gamma=1$, the scheme can be viewed as a dry limit of the Betts-Miller convection scheme (Betts 1986; Betts and Miller 1986). For $\gamma<1$, the scheme mimics the stabilizing effect of latent heat release in moist convection, with the implied latent heat release increasing with decreasing $\gamma$.

We simulated a wide range of circulations with poleequator surface temperature differences in radiative equilibrium, $\Delta_{h}$, between $15 \mathrm{~K}$ and $360 \mathrm{~K}$, resulting in pole-equator surface temperature differences in dy-

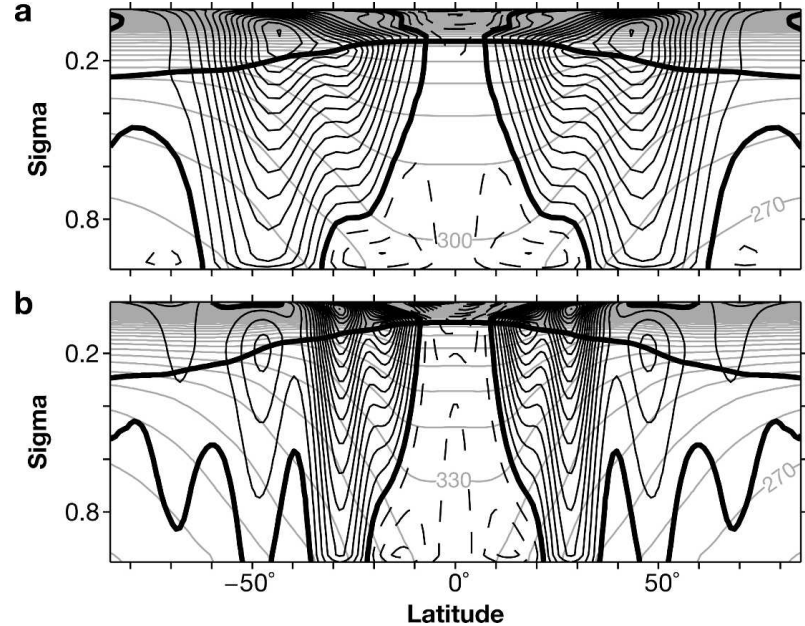

FIG. 2. Mean zonal wind, potential temperature, and tropopause in two GCM simulations. The contour intervals are $10 \mathrm{~K}$ for potential temperature (gray) and $2.5 \mathrm{~m} \mathrm{~s}^{-1}$ for zonal wind (black). The zero zonal wind contour is thick, and negative contours are dashed. The heavy line marks the tropopause. The vertical coordinate is $\sigma=p / p_{s^{*}}$. (a) Simulation with planet radius and rotation rate of Earth $\left(\Delta_{h}=90 \mathrm{~K}, \gamma=0.7\right)$, exhibiting one jet in each hemisphere. (b) Simulation with planet radius of Earth and rotation rate four times that of Earth $\left(\Delta_{h}=120 \mathrm{~K}, \gamma=0.7\right)$, exhibiting a dominant subtropical jet and two weaker extratropical jets in each hemisphere.

namical equilibrium between $12 \mathrm{~K}$ and $166 \mathrm{~K}$. We varied the radii and rotation rates of the simulated planets between the values for Earth and values up to four times larger. We varied convective lapse rates between $5.9 \mathrm{~K} \mathrm{~km}^{-1}(\gamma=0.6)$ and $9.8 \mathrm{~K} \mathrm{~km}^{-1}(\gamma=1.0)$. The simulated circulations include Earth-like circulations and circulations with multiple jets and belts of surface westerlies in each hemisphere-features such as are seen on the giant planets (Fig. 2).

We also calculated the radiative-convective equilibrium states corresponding to the simulated circulations, as reference states for comparisons of how large-scale dynamics modify the thermal structure of the atmosphere. The radiative-convective equilibrium states are independent of the planetary rotation rates and radii (up to a rescaling of the latitude coordinate) and depend only on the convective lapse rate $\gamma \Gamma_{d}$ and on the radiative-equilibrium surface temperature contrast $\Delta_{h}$. The tropospheric lapse rate in radiative-convective equilibrium is equal to the convective lapse rate $\gamma \Gamma_{d}$.

Figure 3 shows extratropical bulk stabilities $\Delta_{v}$ and scaled surface potential temperature gradients $-(f / \beta) \partial_{y} \bar{\theta}_{s}$ in the dynamical equilibria of the GCM simulations and in the corresponding radiative-convective equilibria. The quantities shown are averages over baroclinic zones, with the quantities in the radiativeconvective equilibria averaged in the same way and 

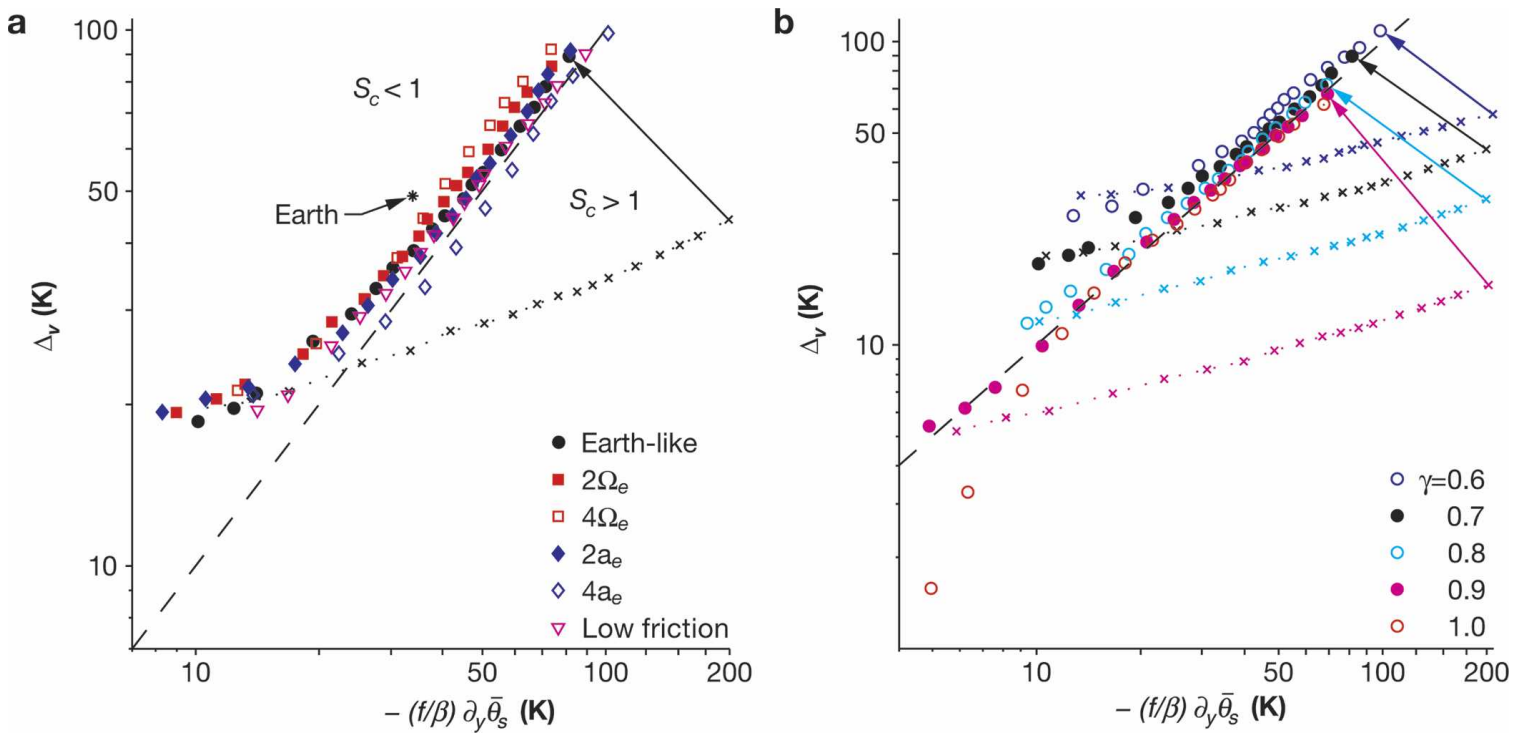

FIG. 3. Extratropical bulk stability $\Delta_{v}$ and scaled surface potential temperature gradient $-(f / \beta) \partial_{y} \bar{\theta}_{s}$ in dynamical equilibria of GCM simulations (plotting symbols defined in legends) and in corresponding radiative-convective equilibria (crosses in colors matching those of the plotting symbols for the dynamical equilibria). (a) Results from Earth-like simulations with terrestrial rotation rate $\Omega_{e}$ and radius $a_{e}$, from simulations with twice and four times the rotation rate and radius of Earth, and from simulations with low frictional drag in the planetary boundary layer (roughness length of surface reduced by a factor of 100). The convective lapse rate is $0.7 \Gamma_{d}=6.8 \mathrm{~K} \mathrm{~km}{ }^{-1}$ in all simulations. (b) Results from simulations with terrestrial rotation rate $\Omega_{e}$ and radius $a_{e}$ and with different convective lapse rates $\gamma \Gamma_{d}(\gamma=0.6, \ldots, 1.0)$. The series of simulations with $\gamma=0.7$ in (b) is the same as the series labeled Earth-like in (a). For each set of parameters, the figure shows a series of simulations obtained by varying the radiative-equilibrium surface temperature contrast $\Delta_{h}$. Displayed quantities are averages over baroclinic zones, averaged in the same way and over the same regions in the dynamical equilibria and in the corresponding radiative-convective equilibria (see appendix $\mathrm{C}$ ). Here and in subsequent figures, the corresponding quantities for Earth's atmosphere (annual means according to reanalysis data averaged over both hemispheres) are shown for comparison (see appendix C). The dashed lines represent supercriticality $S_{c}=1$, with $S_{c}<1$ above it and $S_{c}>1$ below it. For sufficiently large surface potential temperature gradients, all simulations condense onto the line $S_{c} \sim 1$.

over the same regions as the quantities in the corresponding dynamical equilibria. ${ }^{4}$ Only the radiativeconvective equilibria for the Earth-like simulation series are shown in Fig. 3a. The radiative-convective equilibria for the other simulation series in Fig. 3a are identical to those for the Earth-like simulation series. The radiative-convective bulk stabilities and scaled surface potential temperature gradients for the other simulation series differ from those for the Earth-like simulation series only through differences in averaging regions (which do not lead to qualitative differences to the Earth-like simulation series). For the simulations for $\gamma=1$ (dry convection), the radiative-convective bulk stabilities are zero and hence are not shown in Fig. $3 b$. The radiative-convective bulk stabilities increase

\footnotetext{
${ }^{4}$ See appendix $\mathrm{C}$ for the estimation of flow statistics and for the choice of averaging regions. There is some ambiguity in the choice of averaging regions; however, as discussed in appendix $\mathrm{C}$, different choices of averaging regions affect $O(1)$ constants in the scaling relations shown here and in what follows, but they do not substantially affect the scaling relations themselves.
}

with radiative-equilibrium surface temperature contrast $\Delta_{h}$ because the mean surface temperature in radiative equilibrium increases with $\Delta_{h}$, which results in a decrease of the density at the surface and in an increase of the tropopause height in accordance with the radiative constraint on the tropopause height in the idealized GCM (cf. Held 1982; Schneider 2006) — both factors that, given a fixed lapse rate, lead to an increase of bulk stabilities with $\Delta_{h}$. The arrows indicate how the thermal structures in the dynamical equilibria of the GCM simulations with the largest radiative-equilibrium surface temperature contrast $\Delta_{h}=360 \mathrm{~K}$ are modified compared with the thermal structures in the corresponding radiative-convective equilibria.

Figure 3 shows that in simulations with small scaled surface potential temperature gradients $-(f / \beta) \partial_{y} \bar{\theta}_{s}$, the extratropical bulk stability $\Delta_{v}$ is close to the radiativeconvective bulk stability and $S_{c}<1$. (Small reductions of bulk stabilities in dynamical equilibrium compared with radiative-convective equilibrium are probably caused by destabilizing mixing in the planetary boundary layer.) When the scaled surface potential tempera- 

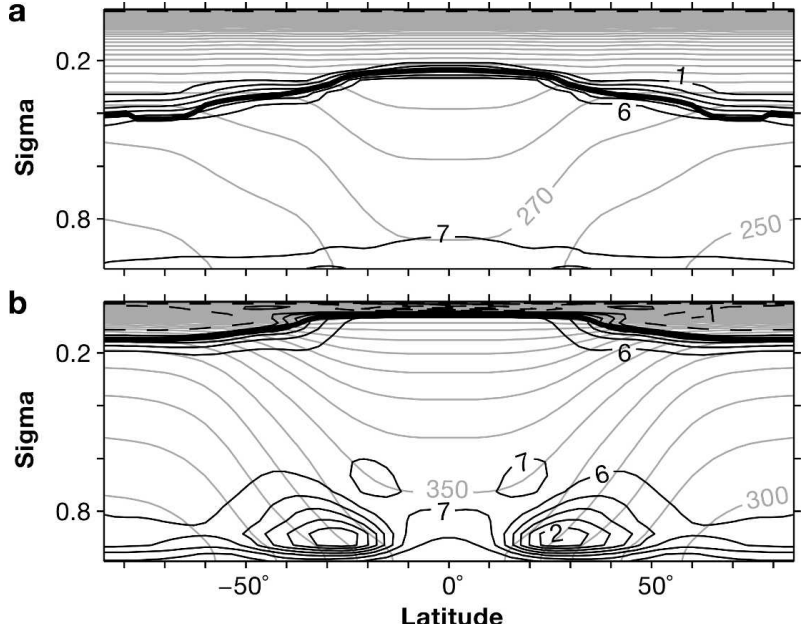

FIG. 4. Temperature lapse rate, potential temperature, and tropopause in two GCM simulations with terrestrial rotation rate and radius and convective lapse rate $0.7 \Gamma_{d}=6.8 \mathrm{~K} \mathrm{~km}^{-1}$. Radiative-equilibrium surface temperature contrasts $\Delta_{h}$ are (a) $30 \mathrm{~K}$ and (b) $180 \mathrm{~K}$. The contour intervals are $10 \mathrm{~K}$ for potential temperature (gray) and $1 \mathrm{~K} \mathrm{~km}^{-1}$ for lapse rate (black, negative contours dashed). The heavy line marks the tropopause. In (a), the lapse rate throughout the troposphere is approximately equal to the convective lapse rate. In (b), the extratropical lapse rate is smaller than the convective lapse rate, particularly near the surface. The greater tropopause height in (b) is primarily due to an increase of the mean surface temperature with radiativeequilibrium surface temperature contrast $\Delta_{h}$.

ture gradients $-(f / \beta) \partial_{y} \bar{\theta}_{s}$ increase beyond the value at which $S_{c} \sim 1$ for a radiative-convective thermal stratification, the bulk stabilities $\Delta_{v}$ increase proportionately, such that $S_{c} \sim 1$. The larger the convective lapse rate, the smaller is the scaled surface potential temperature gradient $-(f / \beta) \partial_{y} \bar{\theta}_{s}$ at which the line $S_{c} \sim 1$ is reached (Fig. 3b). Consistent with theory, for sufficiently large surface temperature gradients, all simulations condense onto the line $S_{c} \sim 1$.

In the regime $S_{c}<1$, the extratropical thermal stratification and tropopause height are set by radiation and convection. The reduction of scaled surface potential temperature gradients in the dynamical equilibria compared with the radiative-convective equilibria is moderate, and the tropospheric lapse rate is approximately equal to the convective lapse rate (see Fig. 4a for an example). In the regime $S_{c} \sim 1$, baroclinic eddies significantly reduce the scaled surface potential temperature gradients, stabilize the extratropical thermal stratification, and modify the tropopause height. The stabilization of the thermal stratification is concentrated near the surface (see Fig. 4b for an example), but for sufficiently large surface temperature gradients or large convective lapse rates, the stratification throughout the troposphere is stabilized by eddies. However, convec- tion only ceases to provide a significant portion of the dynamical heating in the upper troposphere for larger convective lapse rates $(\gamma \geq 0.8)$ and for the largest surface potential temperature gradients we simulated.

Figure $3 \mathrm{~b}$ shows that, consistent with the theory not making reference to a convective or other external lapse rate, bulk stabilities for sufficiently large surface temperature gradients do not depend explicitly on the convective lapse rate (at least not for $\gamma \geq 0.7$ ). This is the case despite large variations of the circulations with convective lapse rates. For example, the strength of the tropical Hadley circulation varies by about a factor of 8 across simulations with comparable scaled surface potential temperature gradients $-(f / \beta) \partial_{y} \bar{\theta}_{s}$ but with different convective lapse rates. A few simulations for $\gamma=$ 1.0 with small surface temperature gradients exhibit supercriticalities greater than one. These large supercriticalities appear to be spurious, caused by small or vanishing near-surface static stabilities. When supercriticalities are estimated with bulk stabilities based on tropospheric averages [as in Eq. (A6)] in place of nearsurface averages, the supercriticalities are similar to or less than one. (Other results do not change substantially if bulk stabilities are based on tropospheric averages in place of near-surface static stabilities.)

\section{Inverse energy cascade and eddy scales}

\section{a. Theory}

The result that the supercriticality does not significantly exceed one implies that there is no inverse energy cascade beyond the scale of the linearly most unstable baroclinic waves. The scale of the linearly most unstable baroclinic waves is the inner Rossby radius

$$
L_{R} \sim \frac{N_{p}\left(\bar{p}_{s}-\bar{p}_{t}\right)}{f} S_{c}
$$

where $N_{p}^{2}=-\left(\bar{\rho}_{s} \bar{\theta}_{s}\right)^{-1}{\overline{\partial_{p}}}^{s}$ is a static stability measure and $\rho$ is the density. The first factor on the right-hand side is an outer Rossby radius; the second factor, the supercriticality $S_{c} \sim\left(\bar{p}_{s}-\bar{p}_{e}\right) /\left(\bar{p}_{s}-\bar{p}_{t}\right)$, accounts for the fact that the effective pressure range $\bar{p}_{s}-\bar{p}_{e}$ of baroclinic waves may be smaller than the outer pressure range $\bar{p}_{s}-\bar{p}_{t}\left(\right.$ Held 1978; Lindzen and Farrell 1980b). ${ }^{5}$

\footnotetext{
${ }^{5}$ We chose to define the Rossby radius as a function of the near-surface static stability for consistency with the near-surface static stability entering the supercriticality and with the scaling arguments that follow; however, defining the Rossby radius, more traditionally, as a function of a tropospheric average of the static stability [as in Eq. (D2)] does not substantially affect the simulation results discussed below (cf. appendix D).
} 
Unless arrested at a smaller scale by dissipation or by the limited size of the planet, an inverse cascade of barotropic eddy kinetic energy would extend from the inner Rossby radius to the Rhines scale (Rhines 1975)

$$
L_{\beta} \sim \frac{\mathrm{EKE}_{\mathrm{bt}}^{1 / 4}}{\beta^{1 / 2}},
$$

where $\mathrm{EKE}_{\mathrm{bt}}$ is the average barotropic eddy kinetic energy per unit mass. Rossby radius and Rhines scale can be related by assuming that the barotropic eddy kinetic energy scales with the available potential energy of the mean flow (MAPE) and some power of the supercriticality,

$$
\mathrm{EKE}_{\mathrm{bt}} \sim \mathrm{MAPE} \times S_{c}^{r} \text {. }
$$

For the exponent, theories based on quasigeostrophic layer models suggest $r=1$ in the weakly nonlinear limit (Pedlosky 1970) and $r=2$ in the strongly nonlinear limit (Held and Larichev 1996); our simulations suggest $r \approx 4 \pm 1$ (and possibly greater for the smallest supercriticalities, for which we only have a few simulations). Combining the Rossby radius (5) and Rhines scale (6) with the estimate (found to be accurate in the simulations)

$$
\mathrm{MAPE} \sim\left(\frac{\partial_{y} \bar{\theta}_{s} L_{e}}{\bar{\rho}_{s} \bar{\theta}_{s} N_{p}}\right)^{2}
$$

for the mean available potential energy per unit mass in a zone of meridional width equal to the energycontaining scale $L_{e}$, we obtain $L_{\beta}^{2} \sim L_{R} L_{e} \times S_{c}^{r / 2}$. Depending on whether the energy-containing scale is similar to the Rossby radius or to the Rhines scale, it follows that

$$
\frac{L_{\beta}}{L_{R}} \sim\left\{\begin{array}{lll}
S_{c}^{r / 4} & \text { if } & L_{e} \sim L_{R} \\
S_{c}^{r / 2} & \text { if } & L_{e} \sim L_{\beta}
\end{array} .\right.
$$

In either case and independent of the value of the exponent $r \geq 0$, the result that the supercriticality does not significantly exceed one implies that the Rhines scale is smaller than or at most similar to the Rossby radius, that is, that an inverse energy cascade beyond the scale of the linearly most unstable waves is inhibited. The inhibition of an inverse energy cascade beyond the scale of the linearly most unstable waves implies that the nonlinear eddy-eddy interactions that would engender an inverse cascade are weak.

\section{b. Simulations}

The simulations with the idealized GCM confirm the theory. The energy-containing scale of barotropic eddy kinetic energy is generally similar to the Rossby radius
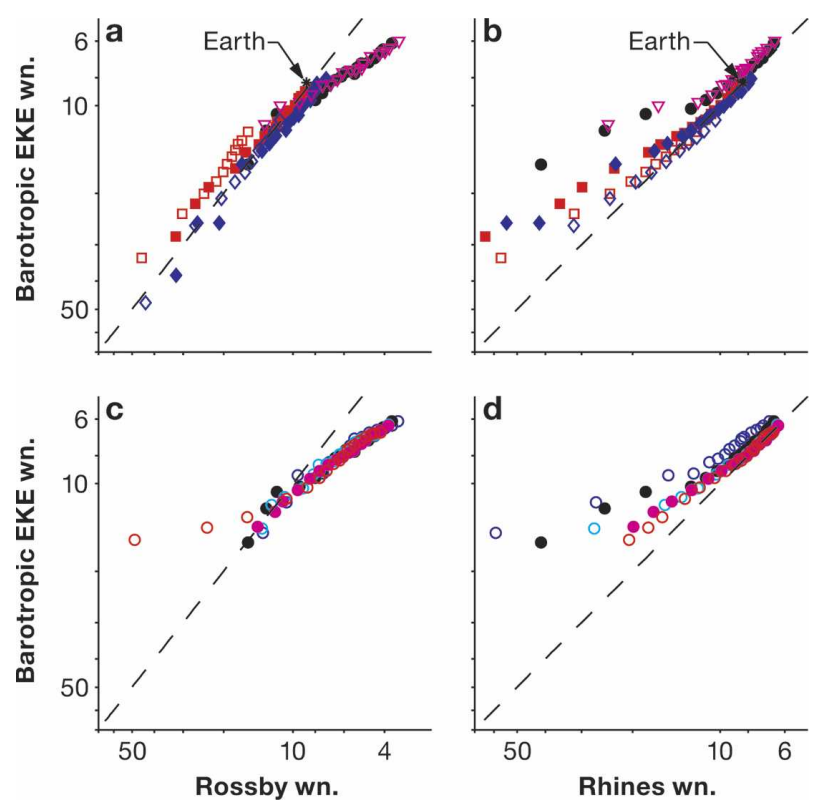

FIG. 5. Energy-containing scale of barotropic eddy kinetic energy vs extratropical Rossby radius (a), (c) and vs Rhines scale (b), (d). Length scales are expressed as spherical wavenumbers (see appendix D). The dashed lines represent the diagonals along which the wavenumbers on the abscissa and ordinate are equal. The energy-containing scale is generally similar to the Rossby radius, except at the largest scales, at which the width of the baroclinic zone appears to limit the scales of baroclinic instability and of the energy-containing eddies. The Rhines scale approaches the energy-containing scale from below as the supercriticality approaches one. The scales for Earth's atmosphere, shown for comparison, are the same as those indicated in Fig. 1. Plotting symbols in (a), (b) as in Fig. 3a, and in (c), (d) as in Fig. 3b.

(Figs. 5a,c; see appendix D for definitions of eddy scales). ${ }^{6}$ An exception are the largest scales, where the width of the baroclinic zone appears to limit the scales of baroclinic instability and of the energy-containing eddies, making the energy-containing scale smaller than the Rossby radius. Anomalously small Rossby radii for $\gamma=1.0$ and small surface temperature gradients (Fig. 5c) are caused by vanishing or small near-surface static stabilities; the anomalies disappear when [as in

\footnotetext{
${ }^{6}$ For different series of simulations, the constants of proportionality relating the energy-containing scale and the Rossby radius differ slightly, as seen at larger wavenumbers $(n \geq 10)$ in Fig. 5a. The variations in proportionality constants are caused by variations of the saturation value of the supercriticality in the different series of simulations (cf. Fig. 3). Rescaling the supercriticalities, for example, by dividing the supercriticalities for each series of simulations by the supercriticality of the simulation with the largest surface temperature gradient, leads to a collapse of the simulations onto the line of equal energy-containing scale and Rossby radius.
} 

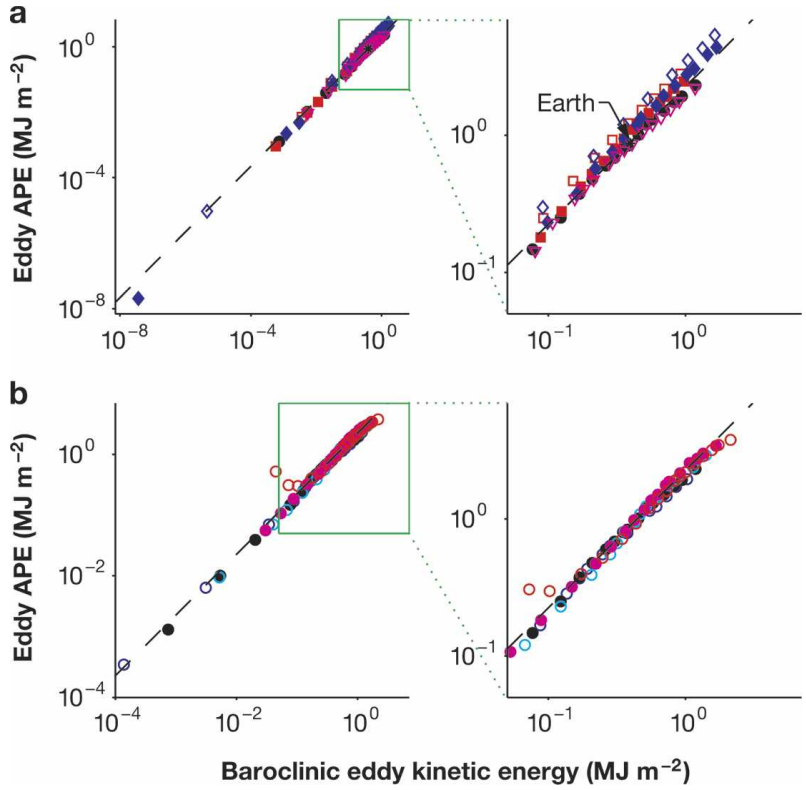

FIG. 6. Eddy available potential energy vs baroclinic eddy kinetic energy. As in the linearly most unstable baroclinic waves, eddy available potential energy and baroclinic eddy kinetic energy are equipartitioned. The dashed line represents the linear relation EAPE $=2.25 \mathrm{EKE}_{\mathrm{bc}}$. Plotting symbols are the same as in Fig. 3.

Eq. (D2)] a tropospheric average of the static stability is used in place of the near-surface static stability in the Rossby radius (5). (Other results again do not change substantially if tropospheric averages of the static stability are used in place of near-surface static stabilities.) For simulations with $S_{c}<1$, the Rhines scale is smaller than the energy-containing scale. The Rhines scale approaches the energy-containing scale from below as the supercriticality approaches one (Figs. 5b,d).

The energy-containing scale in the simulations varies between about wavenumbers 50 and 6 , which excludes the possibility of the planet size generally limiting the inverse energy cascade. The simulations include planets larger and rotating more rapidly than Earth and a planet with low surface friction-conditions that should favor an inverse energy cascade. Several simulations exhibit multiple extratropical jets in each hemisphere (Fig. 2b), with a separation scale between the jets similar to the energy-containing scale (Panetta 1993) and to the Rossby radius, without evidence of an inverse energy cascade beyond the scale of the linearly most unstable waves.

\section{Eddy energies}

The modification of the atmospheric thermal structure by macroturbulence inhibits the inverse energy cascade and renders nonlinear eddy-eddy interactions weak. Hence, although some nonlinear eddy-eddy interactions, such as between the linearly most unstable baroclinic waves and shorter waves (Boer and Shepherd 1983; Shepherd 1987), are doubtlessly important, the scales and structures of the energy-containing eddies resemble those of the linearly most unstable waves.

In the linearly most unstable baroclinic waves, baroclinic eddy kinetic energy and eddy available potential energy are equipartitioned. Over eight decades of eddy energies, the simulations clearly exhibit this equipartitioning (Fig. 6). Anomalously large eddy available potential energies for $\gamma=1.0$ and small surface temperature gradients (Fig. 6b) are again caused by vanishing or small near-surface static stabilities; the anomalies disappear when levels below $\sigma=0.75$ are excluded from the computation of the average eddy available potential energy. If there were an inverse energy cascade beyond the scale of the linearly most unstable waves, the eddy available potential energy (and the barotropic eddy kinetic energy) would be greater than the baroclinic eddy kinetic energy and would increase with the extent of the inverse cascade (Held and Larichev 1996). ${ }^{7}$

It is remarkable that the eddy energies scale linearly with each other and, moreover, that the constant of proportionality relating them appears to be nearly universal for Earth-like circulations $(\sim 2.25)$. The constant of proportionality exhibits only a dependence on planet radius and rotation rate (Fig. 6). The equipartitioning of eddy energies confirms our results without making reference to mean fields, whose definition may be ambiguous.

\section{Discussion and broader implications}

We have shown that large-scale averages of atmospheric thermal structures satisfy $S_{c} \lesssim 1$, implying that the energy-containing scale is similar to the scale of the linearly most unstable waves, nonlinear eddy-eddy interactions are weak, and an inverse energy cascade is inhibited. Theoretical considerations show that if eddyeddy interactions were strongly nonlinear and would engender an inverse energy cascade, baroclinic entropy fluxes would modify the atmospheric thermal structure such that $S_{c} \sim 1$, which means that the strongly nonlinear limit is unattainable. If eddy-eddy interactions are

\footnotetext{
${ }^{7}$ Eddy available potential energy, total eddy kinetic energy, and barotropic eddy kinetic energy are likewise equipartitioned in the simulations; however, unlike that seen in Fig. 6, this equipartitioning would also be possible in strongly nonlinear macroturbulence (Held and Larichev 1996).
} 
weakly nonlinear, the theory suggests that the atmospheric thermal structure satisfies $S_{c} \lesssim 1$, provided the mixing effected by the eddies has no essential vertical structure. Simulations with an idealized GCM confirmed that interactions between eddies and the mean flow lead to self-organization of atmospheric macroturbulence into critical states with $S_{c} \lesssim 1$ in large-scale averages.

Although the constraint $S_{c} \lesssim 1$ structurally resembles quasigeostrophic baroclinic-adjustment constraints on the atmospheric thermal structure (e.g., Stone 1978; Lindzen and Farrell 1980a; Held 1982; Lindzen 1993), it differs from those fundamentally in the theoretical reasoning underlying it. Unlike quasigeostrophic baroclinic-adjustment constraints, the constraint $S_{c} \lesssim 1$ neither presupposes nor implies that the atmosphere is neutral with respect to baroclinic instability. For example, potential vorticity gradients are not assumed to be small in the theory and generally are not small in the simulations; rather, they are sufficiently large that, according to the balance condition (1), isentropic mass fluxes associated with the eddy flux of potential vorticity along isentropes and with the eddy flux of surface potential temperature balance each other upon vertical integration. See Schneider $(2004,2005)$ and appendix A for further comparisons with quasigeostrophic theory.

Interactions between macroturbulence and latent heat release in phase changes of water were not taken into account in the theory and only to a limited extent in the simulations (in the convection scheme). Nonetheless, the simulations with different convective lapse rates, mimicking different degrees of latent heating, and the surprisingly close agreement of Earth's eddy scales and energies with theory and simulations (Figs. 1, $5,6)$ suggest that our results may be generalizable to moist atmospheres. The seasonal (Stone and Nemet 1996) and decadal (Schneider 2004) variability of Earth's extratropical atmosphere satisfies the constraint $S_{c} \lesssim 1$ or similar constraints (Stone 1978), despite considerable variability of the thermal structure. And the fundamental feedback mechanism underlying our results-macroturbulence reduces the surface potential temperature gradient, stabilizes the thermal stratification, and thus limits the nonlinearity of eddyeddy interactions - also acts in a moist atmosphere.

The theory presented here issues from the balance condition (1) between eddy fluxes of potential vorticity and surface potential temperature, a balance condition that derives from the zonal momentum balance and is unaffected by moist processes as long as the Rossby number is small and moist convection does not lead to significant vertical momentum fluxes. However, the central assumption leading to the estimate of the pres- sure range (2) over which baroclinic eddies stabilize the thermal stratification-the assumption that macroturbulence mixes potential vorticity along isentropes and potential temperature along the surface with a vertically essentially uniform eddy diffusivity-may not be adequate for moist atmospheres in general, although it may be justifiable for Earth's atmosphere. Potential vorticity and surface potential temperature are not materially conserved in adiabatic displacements of air masses if the air masses become saturated during a displacement and latent heat is released in phase changes of water. A general theory for moist atmospheres must build upon modified assumptions about mixing of adiabatically conserved quantities by macroturbulence and may lead to a generalized definition of supercriticality. Nonetheless, simulations with an idealized GCM with a hydrological cycle suggest that in moist atmospheres, baroclinic eddies also stabilize the thermal stratification such that an inverse energy cascade is inhibited ( $P$. O'Gorman 2005, personal communication).

The theory and simulations point to fundamental constraints on the global circulations of Earth and other planets. To the extent that the results carry over to moist atmospheres, they constrain the climate changes that are possible on Earth. For example, they constrain the changes in extratropical tropopause pressure that are possible given changes in surface potential temperatures and their gradients (Santer et al. 2003; Schneider 2004). The results should also apply to other differentially heated planets. For example, although the absence of a solid surface on Jupiter makes the interpretation of surface quantities in the theory ambiguous, the results are consistent with what is known about eddy scales and the large-scale structure of Jupiter's circulation. On Jupiter, the separation scale between off-equatorial jets and estimates of the (outer) Rossby radius are of the same order of magnitude $(\sim 2000 \mathrm{~km}$; Ingersoll and Kanamori 1995; Ingersoll et al. 2004), as in our simulations, consistent with the similarity of the separation scale to the energy-containing scale and to the Rossby radius. There may be an inverse energy cascade on Jupiter from small convective scales to the Rossby radius (Ingersoll et al. 2000), but apparently not beyond it.

The demonstrated importance of feedbacks between baroclinic eddies and the thermal stratification implies that two-dimensional and quasigeostrophic turbulence are inadequate paradigms for understanding atmospheric macroturbulence.

Acknowledgments. We are grateful for support by the Davidow Discovery Fund, by an Alfred P. Sloan Research Fellowship, and by the National Science 
Foundation (Grant ATM-0450059); for computing resources provided by the National Center for Atmospheric Research (which is sponsored by the National Science Foundation); and for comments on drafts of this paper by Paul O'Gorman and Tim DelSole. The program code for the simulations described in this paper, as well as the simulation results themselves, are available from the authors upon request.

\section{APPENDIX A}

\section{Vertical Extent of Baroclinic Entropy Fluxes}

\section{a. Estimate for pressure range}

The point of departure for the derivation of the estimate (2) for the pressure range over which baroclinic eddies redistribute entropy is the balance condition (1), a statement of zonal momentum or potential vorticity balance in the temporal and zonal mean in isentropic coordinates (Schneider 2005). The balance condition presumes a hydrostatic ideal-gas atmosphere with stationary flow statistics and with the planet's surface as the only dynamically relevant boundary. Neglected in the balance condition are an Ekman mass flux associated with zonal frictional forces and terms of order Rossby number, such as the cross-isentropic advection of zonal momentum. [Alternatively, one can derive the balance condition from the geostrophic part of the zonal momentum equation; see Schneider (2006).] An additional approximation is involved in obtaining the isentropic mass flux $-\bar{\rho}_{\theta}^{0} \overline{\tilde{v}_{s}^{\prime} \theta_{s}^{s}}$ associated with the geostrophic eddy flux of surface potential temperature from a vertical integral of a surface pressure drag, which the flow along isentropes experiences at intersections of isentropes with the surface. This approximation was found to entail typical midlatitude errors of $10 \%$ and often less (Schneider 2005). The balance condition (1) and the line of argument building upon it depend on conventions for the mean potential vorticity on near-surface isentropes. We have chosen a convention that is convenient for eddy flux closures [convention I of Schneider (2005)], which are our focus here.

Substituting diffusive eddy flux closures $\overline{v^{\prime} P^{\prime}} *$ $-\left.D \partial_{y} \bar{P}^{*}\right|_{\theta}$ and $\overline{\tilde{v}_{s}^{\prime} \theta_{s}^{\prime}} \sim-D \partial_{y} \bar{\theta}_{s}$ into the balance condition (1) and assuming that the eddy diffusivity $D$ has no essential vertical structure below the upper bound $\theta_{e}$ of the integration, one can take the diffusivity outside the integral and finds

$$
\int_{\theta_{b}}^{\theta_{e}} \frac{\left.\bar{\rho}_{\theta} \partial_{y} \bar{P}^{*}\right|_{\theta}}{\bar{P}^{*}} d \theta \sim-\bar{\rho}_{\theta}^{0} \partial_{y} \bar{\theta}_{s} .
$$

To estimate the integral on the left-hand side, we use the small Rossby number (or planetary-geostrophic) approximation $\bar{P}^{*} \sim f / \bar{\rho}_{\theta}$ for the mean potential vorticity and the relation $\bar{\rho}_{\theta}=-g^{-1} \partial_{\theta} \bar{p}$ between isentropically averaged pressure $\bar{p}$ and isentropic density $\bar{\rho}_{\theta}$, together with the convention that the pressure is equal to the surface pressure and the isentropic density vanishes on isentropes with potential temperature less than the instantaneous surface potential temperature (Lorenz 1955). This leads to

$$
\begin{aligned}
\int_{\theta_{b}}^{\theta_{e}} \frac{\left.\bar{\rho}_{\theta} \partial_{y} \bar{P}^{*}\right|_{\theta}}{\bar{P}^{*}} d \theta & \sim \int_{\theta_{b}}^{\theta_{e}}\left(\frac{\beta}{f} \bar{\rho}_{\theta}-\partial_{y} \bar{\rho}_{\theta}\right) d \theta \\
& \sim \frac{\beta}{f} \frac{\bar{p}_{s}-\bar{p}_{e}}{g}+\frac{\left.\partial_{y} \bar{p}\right|_{\theta=\theta_{e}}}{g},
\end{aligned}
$$

where $\bar{p}_{s}=\bar{p}\left(\theta_{b}\right)$ is the mean surface pressure and $\bar{p}_{e}=$ $\bar{p}\left(\theta_{e}\right)$ is the mean pressure at the isentrope with potential temperature $\theta_{e}$. (The mean surface pressure arises from Lorenz's convention of setting the pressure on isentropes with potential temperature less than the instantaneous surface potential temperature to the surface pressure, which implies that the mean pressure on isentropes at the nominal lower boundary $\theta_{b}$ is equal to the mean surface pressure.) Neglected on the righthand side is the boundary term $-\left.g^{-1} \partial_{y} \bar{p}\right|_{\theta=\theta_{b}}=$ $-g^{-1} \partial_{y} \bar{p}_{s}$, which is generally much smaller than the other terms on the right-hand side (Schneider 2004).

The developments up to this point are discussed in greater detail by Schneider (2004). What follows extends the developments and improves the estimates of Schneider (2004).

One obtains an estimate for the pressure $\bar{p}_{e}$ up to which baroclinic eddies redistribute entropy by combining Eqs. (A1) and (A2) and by using an additional approximation for the mean isentropic density $\bar{\rho}_{\theta}^{0}=\bar{\rho}_{\theta}\left(\bar{\theta}_{s}\right)$ at the mean surface potential temperature. With the Heaviside step function $\mathcal{H}(\cdot)$ indicating that the isentropic density vanishes on isentropes with potential temperature less than the instantaneous surface potential temperature $\theta_{s}(x, y, t)$, we can write the isentropic density at the mean surface potential temperature as

$$
\begin{aligned}
\bar{\rho}_{\theta}^{0} & =\overline{\rho_{\theta}\left(\bar{\theta}_{s}\right) \mathcal{H}\left(\bar{\theta}_{s}-\theta_{s}\right)} \\
& =\overline{\rho_{\theta}\left(\theta_{s}-\theta_{s}^{\prime}\right) \mathcal{H}\left(\bar{\theta}_{s}-\theta_{s}\right)} \\
& \sim \overline{\rho_{\theta}\left(\theta_{s}\right)} \overline{\mathcal{H}\left(\bar{\theta}_{s}-\theta_{s}\right)} \\
& \sim-\left(2 g \overline{\partial_{p} \theta}\right)^{-1} .
\end{aligned}
$$

In the last two lines, we have neglected vertical variations of isentropic density near the surface and fluctuations at the surface and have assumed that the median surface potential temperature is approximately equal to the mean surface potential temperature, such that 
$\overline{\mathcal{H}\left(\bar{\theta}_{s}-\theta_{s}\right)} \approx 0.5$ (Schneider 2005). Using the approximation (A3) along with the relation $\left.\partial_{y} \bar{p}\right|_{\theta} \sim$ $-\left.\left(\partial_{p} \bar{\theta}^{p}\right)^{-1} \partial_{y} \bar{\theta}^{p}\right|_{p(\theta)}$, where $\overline{(\cdot)}^{p}$ denotes the temporal and zonal mean along isobaric surfaces, and combining Eqs. (A1) and (A2) yields

$$
\bar{p}_{s}-\bar{p}_{e} \sim \frac{f}{\beta} \frac{\partial_{y} \bar{\theta}_{s}}{2 \bar{\partial}_{p} \theta}\left(1+2 \frac{I_{e}}{I_{s}}\right),
$$

where

$$
I_{s}=\frac{\partial_{y} \bar{\theta}_{s}}{\partial_{p} \theta} \quad \text { and } \quad I_{e}=\left.\frac{\partial_{y} \bar{\theta}^{p}}{\left.\overline{\partial_{p} \theta}\right|_{p} ^{p}}\right|_{p=\bar{p}_{e}}
$$

are measures of the slope of isentropes at the surface and at the pressure $\bar{p}_{e}$ up to which baroclinic eddies redistribute entropy.

The second term in parenthesis, $2 I_{e} / I_{s}$, goes back to the pressure gradient term on the right-hand side of Eq. (A2) and is often small. If baroclinic eddies redistribute entropy up to the tropopause $\left(\bar{p}_{e} \sim \bar{p}_{t}\right.$ or $\left.S_{c} \sim 1\right)$, this term is generally much less than one, because, at the tropopause, the absolute value of the meridional potential temperature gradient is generally smaller and the absolute value of the static stability factor $-\partial_{p} \theta=$ $g^{-1} \partial_{z} \theta / \rho$ is generally greater than at the surface. In those of our simulations in which $S_{c} \sim 1$, the term $2 I_{e} / I_{s}$ is less than 0.10 for $\bar{p}_{e}=\bar{p}_{t}$ (with surface averages interpreted as near-surface averages, as described in appendix C); it is less than 0.05 in the simulations with the largest surface temperature gradients. In Earth's atmosphere, the term $2 I_{e} / I_{s}$ is about 0.04 for $\bar{p}_{e}=\bar{p}_{t}$. In simulations with small supercriticalities, the term $2 I_{e} / I_{s}$ is typically less than 0.15 for $\bar{p}_{e}=\bar{p}_{t}$, except for a few simulations with small convective lapse rate $(\gamma=0.6)$, in which it can be as large as 0.24 ; it may be somewhat greater at the (higher) actual pressure $\bar{p}_{e}$ up to which baroclinic eddies redistribute entropy. We neglect the term $2 I_{e} / I_{s}$, although this may lead to a slight underestimation of the vertical extent of entropy fluxes, in particular for small supercriticalities. Thus, the estimate (A4) leads to the estimate (2) for the pressure up to which baroclinic eddies redistribute entropy.

Neglecting the term $2 I_{e} / I_{s}$ or the pressure gradient term on the right-hand side of Eq. (A2) amounts to assuming that the density-weighted logarithmic potential vorticity gradient on the left-hand side of Eq. (A2), in the planetary-geostrophic approximation, integrates to $\beta / f$ times the mass per unit area of that part of the atmospheric column within which baroclinic eddies redistribute entropy. The contribution of the isentropic density gradient to the potential vorticity gradient- the second term on the right-hand side in the first line of
Eq. (A2) - integrates to zero, not because it would generally be small, but because it changes sign between the isentropic-coordinate surface layer and the interior atmosphere (Schneider 2005). This is analogous to the density-weighted logarithmic potential vorticity gradient in the quasigeostrophic two-layer model, which, in the planetary-geostrophic approximation, likewise integrates (sums over the two layers) to $\beta / f$ times the mass per unit area of the two layers. However, unlike in the quasigeostrophic two-layer model, the integral of the isentropic potential vorticity gradient is balanced, according to Eq. (A1), by a term involving the surface potential temperature gradient, which would not appear in a two-layer model. See Schneider $(2004,2005)$ for a more detailed discussion of commonalities and differences between the developments here and quasigeostrophic theory.

Although the balance condition (1) holds for temporal and zonal means latitude-by-latitude, the estimate (2) can only be expected to hold on meridional scales that are similar to or larger than eddy length scales, since it is based on eddy flux closures that can only be expected to hold on such larger scales. The results shown for the simulations and for Earth's atmosphere are therefore averages over extratropical baroclinic zones.

If there is a mixed layer near the surface, such that ${\overline{\partial_{p} \theta}}^{s} \approx 0$, the estimate (2) must be modified. Theory of how a mixed layer couples to the large-scale dynamics of the interior atmosphere is incomplete. As a heuristic, we replace surface averages by averages immediately above the mixed layer [see Held and Schneider (1999) and appendix C].

\section{b. Relation to previous estimates}

Schneider (2004) gives an estimate for the potential temperature $\theta_{e}$ up to which baroclinic eddies redistribute entropy, focusing on a dynamical regime in which the potential temperature $\theta_{e}$ determines the mean tropopause potential temperature $\bar{\theta}_{t}$, such that $\theta_{e} \sim \bar{\theta}_{t}$ and $S_{c} \sim 1$. This estimate can be recovered as a special case of the estimate (2).

With $-{\overline{\partial_{p} \theta}}^{s} \sim g^{-1} \bar{\partial}_{z} \theta / \bar{\rho}^{s} \sim H{\overline{\partial_{z} \theta}}^{s} / \bar{p}_{s}$, where $H=$ $R \bar{T}^{s} / g$ is a scale height, the bulk stability $\Delta_{v}=$ $-2 \overline{\partial_{p} \theta} s\left(\bar{p}_{s}-\bar{p}_{t}\right)$ can be approximated by

$$
\Delta_{v} \sim 2 H \overline{\partial_{z} \theta} s \frac{\bar{p}_{s}-\bar{p}_{t}}{\bar{p}_{s}} .
$$

Estimating the static stability by $\bar{\partial}_{z}{ }^{s} \sim\left(\bar{\theta}_{t}-\bar{\theta}_{s}\right) / H_{t}$, where $H_{t}$ is the mean tropopause height, yields

$$
\Delta_{v} \sim \alpha\left(\bar{\theta}_{t}-\bar{\theta}_{s}\right)
$$


where the nondimensional factor

$$
\alpha=2 \frac{H}{H_{t}} \frac{\bar{p}_{s}-\bar{p}_{t}}{\bar{p}_{s}} \sim 2 \frac{H}{H_{t}}\left(1-e^{-H_{t} / H}\right)
$$

depends on the quotient of tropopause height and scale height. In Earth's atmosphere and in the simulations analyzed by Schneider (2004), the extratropical tropopause height is somewhat greater than the scale height $\left(H_{t} / H \approx 1.40 \pm 0.15\right)$, so that the nondimensional factor is $\alpha \approx 1$. In this case, we recover the estimate of Schneider (2004), valid for $S_{c} \approx 1$,

$$
\Delta_{v} \sim \bar{\theta}_{t}-\bar{\theta}_{s} \sim-\frac{f}{\beta} \partial_{y} \bar{\theta}_{s} .
$$

In some of the simulations presented here, however, the extratropical tropopause height is up to 5 times greater than the scale height, and variations of the nondimensional factor $\alpha$ in the bulk stability (A6) are important in accounting for the simulation results. Moreover, since the static stability $\partial_{z} \theta$ is not constant throughout the troposphere, using a bulk stability (A5) based on the near-surface static stability gives marginally better agreement with the simulation results than using a bulk stability (A6) based on tropospheric averages.

\section{c. Relation to quasigeostrophic estimates}

Within quasigeostrophic theory, Held (1978) offers a similar and similarly motivated estimate for a height scale up to which baroclinic eddies redistribute entropy. The estimate (2) generalizes the quasigeostrophic estimate. Unlike the quasigeostrophic estimate, it does not rely on an assumption of small slopes of isentropes near the surface (Schneider 2005).

Estimates that structurally resemble (2) also arise within quasigeostrophic theory if one considers the pressure range over which baroclinic eddies would need to modify the atmospheric thermal structure to stabilize it with respect to baroclinic instability (Lindzen and Farrell 1980a; Lindzen 1993). These quasigeostrophic baroclinic-adjustment estimates differ from the present estimate in that they assume weak nonlinear eddy-eddy interactions from the outset and in that temperature gradients and static stabilities in the interior atmosphere appear in place of the surface averages in the estimate (2). See Schneider $(2004,2005)$ for the reasons for and implications of this difference. Using nearsurface averages of temperature gradients, rather than averages in the interior atmosphere, is important in accounting for our simulation results; however, using near-surface averages of static stabilities, rather than averages over tropospheric columns as in the bulk sta- bility (A6), affects the results only marginally. As in other simulation studies (Thuburn and Craig 1997), the dynamical equilibria of our simulations are not baroclinically adjusted in any of the ways that have been proposed for obtaining baroclinically neutral states (e.g., Lindzen and Farrell 1980a; Held 1982; Lindzen 1993): neither potential vorticity gradients nor surface potential temperature gradients are generally small. In fact, it is their nonvanishing that gives rise to the estimate (2) and to the appearance of the planetary vorticity gradient $\beta$ and of the surface potential temperature gradient $\partial_{y} \bar{\theta}_{s}$ in it [cf. Eqs. (A1) and (A2)]. See Schneider (2005) for a more detailed discussion of potential vorticity gradients with illustrations from one of the idealized GCM simulations.

\section{APPENDIX B}

\section{Idealized GCM}

The idealized GCM is based on the Flexible Modeling System developed at the Geophysical Fluid Dynamics Laboratory (see online at www.gfdl.noaa.gov $/ \sim \mathrm{fms}$ ). It is a spectral-transform, primitive-equation model in vorticity-divergence form (Bourke 1974), with triangular truncation of the spectral representation. The spherical model surface is spatially uniform and thermally insulating, without topography and with a constant roughness length.

\section{a. Resolution}

Simulations with planet radius and rotation rate equal to those of Earth were performed at horizontal resolution T42, simulations with twice Earth's radius or rotation rate at $\mathrm{T} 85$, and simulations with four times Earth's radius or rotation rate at T127. In all cases, the vertical discretization is based on a centered difference scheme with $30 \sigma$-levels. We performed a simulation with terrestrial parameters at horizontal resolution T106 and simulations with four times Earth's radius and rotation rate at horizontal resolution T85 to verify that resolution does not substantially affect our results.

\section{b. Radiative forcing}

Radiative forcing is represented by Newtonian relaxation of temperatures toward zonally symmetric radiative-equilibrium temperatures $T^{e}$. The temperature fields $T^{e}$ are radiative-equilibrium temperatures of atmospheres transparent to solar radiation and gray for infrared radiation. They are statically unstable in the middle and lower troposphere, in contrast to the stati- 
cally stable equilibrium temperature fields often used in idealized GCMs (e.g., Held and Suarez 1994).

The surface temperature in radiative equilibrium varies with latitude $\phi$ as

$$
T_{s}^{e}(\phi)=\tilde{T}_{s}^{e}+\Delta_{h} \cos ^{2} \phi .
$$

Here, $\tilde{T}_{s}^{e}=260 \mathrm{~K}$ is the surface temperature at the poles, and $\Delta_{h}$ is the pole-equator surface temperature difference, which we varied from 15 to $360 \mathrm{~K}$. The radiative-equilibrium temperature of the overlying atmosphere depends on the surface temperature $T_{s}^{e}$ and on pressure, decreasing with altitude and reaching a constant temperature of $200 \mathrm{~K}$ at the top of the atmosphere. The pressure-dependence of the radiativeequilibrium temperatures is identical to that described by Schneider (2004).

The dependence of the radiative-equilibrium surface temperature on the surface temperature contrast $\Delta_{h}$ differs from that described by Schneider (2004). Here we keep the radiative-equilibrium temperatures at the poles $\tilde{T}_{s}^{e}$ fixed with varying $\Delta_{h}$, thus increasing the global-mean radiative-equilibrium surface temperature $\tilde{T}_{s}^{e}+2 / 3 \Delta_{h}$ with increasing $\Delta_{h}$. Keeping the polar radiative-equilibrium temperatures fixed ensures that, for all values of $\Delta_{h}$, the radiative-equilibrium states near the poles are statically unstable in the middle and lower troposphere, which they would not be for large $\Delta_{h}$ if the global-mean surface temperatures were fixed (cf. Schneider 2004). The increase of global-mean surface temperatures with $\Delta_{h}$ entails a concomitant increase of tropopause height in accordance with the radiative constraint on the tropopause height in the model (Held 1982; Schneider 2006).

Temperatures are relaxed toward radiative equilibrium on a time scale that varies, as described by Schneider (2004), from 7 days near the surface in low latitudes to 50 days in the interior atmosphere.

\section{c. Convection scheme}

Mimicking moist convection in a model in which latent heat release is otherwise not taken into account, a convection scheme relaxes temperatures toward profiles with a specified convective lapse rate if an atmospheric layer is statically unstable relative to the convective lapse rate. The convective lapse rate is $\gamma \Gamma_{d}$, where $\Gamma_{d}=g / c_{p}=9.8 \mathrm{~K} \mathrm{~km}^{-1}$ is the dry adiabatic lapse rate and $\gamma$ is a rescaling parameter.

Convective layers are identified by lifting a parcel from the lowest model level, following a temperature profile with lapse rate $\gamma \Gamma_{d}$. The level of neutral buoyancy at the top of the first region of positive buoyancy that the parcel encounters is the top of the convective layer. The bottom of the convective layer is the lowest model level, regardless of where the parcel first becomes positively buoyant. The presence of a region of positive buoyancy leads to convection only if the convective layer has positive convective available potential energy relative to the lapse rate $\gamma \Gamma_{d}$.

The temperature profile toward which temperatures within a convective layer are relaxed has a lapse rate $\gamma \Gamma_{d}$ and the same vertically integrated enthalpy as the convective layer. Temperatures are relaxed toward the convective profile on a time scale of $4 \mathrm{~h}$.

\section{d. Subgrid-scale dissipation}

Turbulent dissipation in a planetary boundary layer of fixed height $(2.5 \mathrm{~km})$ is represented as vertical diffusion of momentum and dry static energy, with turbulent Prandtl number one (Smagorinsky et al. 1965). The model surface has a constant roughness length of $5 \mathrm{~cm}$. In the series of simulations labeled low friction in the figures, the roughness length is $0.05 \mathrm{~cm}$.

Above the planetary boundary layer, horizontal $\nabla^{8}$ hyperdiffusion in the vorticity, divergence, and temperature equations is the only dissipative process aside from the parameterized radiation. The hyperdiffusion coefficient is chosen such that the smallest resolved scale is damped on a time scale of $12 \mathrm{~h}$.

\section{APPENDIX C}

\section{Estimation of Flow Statistics}

The results shown are averages from statistically stationary states obtained after a spinup period for each simulation. The first simulation was spun up for several thousand simulated days; each subsequent simulation was started from a state nearby in parameter space. Simulations with T42 resolution were run for 300 days; results shown from these simulations are averaged over the final 200 days. Simulations with T85 resolution (twice Earth's radius or rotation rate) were run for 200 days; results are an average of the final 100 days. Simulations with T127 resolution (four times Earth's radius or rotation rate) were run for 250 days; results are an average of the final 150 days (with a day referring to $86400 \mathrm{~s}$, independent of planetary rotation rate). Statistics were estimated from flow fields sampled four times per day. Statistics from the (statistically identical) Northern and Southern Hemisphere were averaged.

\section{a. Extratropical averages}

The results shown are averages over extratropical baroclinic zones whose boundaries are defined as the outermost latitudes at which the meridional eddy flux 
of potential temperature $\overline{v^{\prime} \theta^{\prime}} \sigma \cos (\phi)$ at the nearsurface level $\sigma=0.84$ is $30 \%$ of its maximum value. [The symbol $\overline{(\cdot)}^{\sigma}$ denotes the surface-pressure weighted temporal and zonal mean along $\sigma$-surfaces.] In some simulations, the baroclinic zones encompass multiple jets and belts of surface westerlies (cf. Fig. 2b). For each simulation, we used reference values for the Coriolis parameter $f$ and its gradient $\beta$, defined as the values of the parameters at the latitude of maximum eddy potential temperature flux $\left.\overline{v^{\prime} \theta^{\prime}} \sigma \cos (\phi)\right|_{\sigma=0.84}$.

The latitude of maximum eddy potential temperature flux and the boundaries of the baroclinic zones were determined from cubic smoothing splines fitted to the potential temperature flux. To reduce noise in the results that would be solely due to noise in the determination of averaging regions, within each series of simulations with varying $\Delta_{h}$, we used robust local linear smoothing to ensure smooth variations with $\Delta_{h}$ of the latitudes of the boundaries of the baroclinic zones and of the maxima of the eddy potential temperature flux.

\section{b. Surface averages}

We interpreted all surface averages in the theory as near-surface averages, defined as averages over the layer between $\sigma=0.80$ and 0.70 , which is immediately above the layer in which boundary layer diffusion of dry static energy strongly affects temperatures. An exception is the static stability factor $-{\overline{\partial_{p} \theta}}^{s}$ in the bulk stability (3), for which we used the approximation $-{\overline{\partial_{p} \theta}}^{s} \sim g^{-1}{\overline{\partial_{z} \theta}}^{\sigma} / \bar{\rho}_{s}$, with the average between $\sigma=0.80$ and 0.70 for the static stability $\overline{\partial_{z} \theta} \sigma$ but with the density $\bar{\rho}_{s}$ evaluated at the lowest model level $(\sigma=0.99)$. Evaluating the density at the lowest model level avoids the increase of the estimated static stability factor $-{\overline{\partial_{p}} \theta}^{s}$ with height of the averaging level that is due to the decrease of density with height. This reduces the dependence of the estimated static stability factor $-\bar{\partial}_{p} \theta$ on the height of the averaging level. Evaluating the density at a different level would change the estimated bulk stabilities and supercriticalities by an approximately constant $O(1)$ factor.

\section{c. Determination of tropopause pressure}

The troposphere can be defined as the atmospheric layer within which the bulk of the entropy that the atmosphere receives at the surface is dynamically redistributed (Schneider 2004). The entropy transport from the surface into the interior troposphere, which may be accomplished by baroclinic eddies, convection, or other dynamic processes, is balanced by radiative cooling. To the extent that there are no significant entropy sources in the interior atmosphere, the tropopause is marked by a strong decrease in radiative relaxation rate $c_{r}=$ $\left|\bar{\rho}_{\theta}^{-1} \partial_{\theta}\left(\bar{\rho}_{\theta} \bar{Q}_{r}^{*}\right)\right|$, where $Q_{r}$ is the material potential temperature tendency due to longwave radiation (Newtonian relaxation in our simulations). The time scale $c_{r}^{-1}$ is a replacement time scale of air masses on isentropes (Schneider 2004).

At any given latitude, we determined the tropopause pressure $\bar{p}_{t}$ as the lowest pressure at which the radiative relaxation rate $c_{r}$ is equal to the critical value (60 days $)^{-1}$. In the simulations, this critical value gives well-defined tropopause pressure estimates; choosing other critical values close to the Newtonian relaxation rate in the interior atmosphere, $(50 \text { days })^{-1}$, gives similar tropopause pressures. The tropopause pressure was determined by linear interpolation of radiative relaxation rates $c_{r}$, estimated from averages of heating rates $Q_{r}$ and isentropic densities $\rho_{\theta}=-\left(g \partial_{p} \theta\right)^{-1}$ along $\sigma$-surfaces. This yields tropopause pressures similar to those obtained with the World Meteorological Organization's (WMO) convention of defining the tropopause as a $2 \mathrm{~K} \mathrm{~km}^{-1}$ isoline of the temperature lapse rate. For simulations resembling Earth's climate, the two ways of determining the tropopause give midlatitude tropopause pressures within about $10 \mathrm{hPa}$ of each other, with differences of up to $30 \mathrm{hPa}$ in the subtropics. We prefer using the relaxation rate $c_{r}$ because the transition in relaxation rate $c_{r}$ at the tropopause is sharper than that in lapse rate. Nonetheless, the results shown are essentially unchanged if the tropopause is determined according to the WMO lapse rate criterion.

\section{d. Radiative-convective equilibrium states}

We computed radiative-convective equilibrium states corresponding to the simulated circulations by time-marching the thermodynamic equation with only the parameterized radiation and convection terms until equilibrium was reached. Bulk stabilities and nearsurface potential temperature gradients were computed as in the corresponding dynamical equilibria, using averages over the same baroclinic zones and with the same conventions for near-surface averages. We also used the same reference values for the Coriolis parameter $f$ and its gradient $\beta$ in radiative-convective equilibrium as in dynamical equilibrium. The tropopause pressure in radiative-convective equilibrium was determined as the pressure at which the curvature $\partial_{z z} T$ of the vertical temperature profile is maximal (which is a sharply defined pressure).

\section{e. Eddy energies}

Eddy energies were computed from surface-pressure weighted means along $\sigma$-surfaces and were averaged 
over baroclinic zones. Total eddy kinetic energies $\mathrm{EKE}_{\text {tot }}$ were computed from vertically integrated horizontal wind variances, barotropic eddy kinetic energies $E E_{b t}$ were computed from variances of vertically integrated horizontal velocities, and baroclinic eddy kinetic energies were computed as the difference $\mathrm{EKE}_{\mathrm{bc}}$ $=\mathrm{EKE}_{\mathrm{tot}}-\mathrm{EKE}_{\mathrm{bt}}$.

Eddy available potential energies were estimated, in the manner described by Lorenz (1955), from potential temperature variances on $\sigma$-surfaces. Levels below $\sigma=$ 0.9 and above $\sigma=0.025$ were not taken into account in the estimation of eddy available potential energies to avoid artifacts due to boundary layer diffusion and due to the rigid-lid upper boundary of the model (at $\sigma=0$ ).

\section{f. Sensitivity of results to choice of averaging regions}

The results shown do not change substantially if averaging regions and surface averages are defined differently. For example, evaluating surface averages on different levels near the surface does not change the fact that, for sufficiently large surface temperature gradients, the simulations condense onto lines with nearly constant supercriticalities $S_{c} \sim 1$. However, the values of the $O(1)$ supercriticality constants depend on the level at which surface averages are evaluated; the constants differ by up to about $10 \%$ for different plausible choices of averages (except for simulations with $\gamma=1.0$ and small surface temperature gradients; see sections 2 and 3). The results shown also do not change substantially if baroclinic zones are defined differently, for example, by defining their boundaries as the outermost latitudes at which the meridional eddy flux of potential temperature $\left.\overline{v^{\prime} \theta^{\prime}} \sigma \cos (\phi)\right|_{\sigma=0.84}$ is $15 \%$ or $75 \%$ in place of $30 \%$ of its maximum value. The scaling of eddy energies is insensitive to the choice of averaging regions, since eddy available potential energy and eddy kinetic energy are concentrated in the baroclinic zones. The linear scaling relations between eddy available potential energy and eddy kinetic energy suggested by Fig. 6 are unchanged if eddy energies are averaged globally rather than over baroclinic zones.

\section{g. Statistics for Earth's atmosphere}

The quantities for Earth's atmosphere included in the figures were computed from reanalysis data for the years 1980-2001 provided by the European Centre for Medium-Range Weather Forecasts (ERA-40 reanalyses: T159 horizontal resolution, 60 vertical levels; see Kållberg et al. 2004). The quantities shown are annual means over both hemispheres, averaged in the same manner as the corresponding quantities for the simula- tions. The only difference between the flow statistics for the reanalysis data and for the simulations is that, for the reanalysis data, the tropopause pressure was determined according to the WMO convention as a $2 \mathrm{~K} \mathrm{~km}^{-1}$ isoline of the temperature lapse rate. Eddy fields for reanalysis data are fluctuations about annual and zonal means and thus include contributions from stationary eddies. We chose the same $O(1)$ constants entering the characteristic length scales for Earth's atmosphere as for the simulations (see appendix D).

Annual mean statistics for the Northern and Southern Hemisphere individually are likewise consistent with the simulation results, as are statistics for different seasons.

\section{APPENDIX D}

\section{Energy-Containing Scale, Rossby Radius, and Rhines Scale}

The wavenumbers in Figs. 1 and 5 are nondimensional spherical wavenumbers $n$, defined so that $-n(n+1) / a^{2}$ are eigenvalues of the Laplacian on a sphere of radius $a$ (Boer 1983).

\section{a. Energy-containing scale}

Based on the wavenumber spectrum $E_{n}$ of barotropic eddy kinetic energy - that is, the wavenumber spectrum of barotropic kinetic energy, computed as described by Boer and Shepherd (1983), but without the zonal-wavenumber zero component-we define the energycontaining wavenumber $n_{e}$ as the wavenumber for which

$$
n_{e}\left(n_{e}+1\right)=\frac{\sum_{n} E_{n}}{\sum_{n}[n(n+1)]^{-1} E_{n}} .
$$

Since the barotropic eddy kinetic energy can be expressed in terms of the horizontal streamfunction $\Psi^{\prime}$ of barotropic eddy velocities as $\left\langle\mathrm{EKE}_{\mathrm{bt}}\right\rangle=-\left\langle\Psi^{\prime} \nabla^{2} \Psi^{\prime}\right\rangle / 2=$ $\sum E_{n}$, where $\langle\cdot\rangle$ denotes a global average, and since $\left\langle\Psi^{\prime 2}\right\rangle / 2=a^{2} \sum[n(n+1)]^{-1} E_{n}$ by inversion of the Laplacian, this definition of energy-containing wavenumber is equivalent to the definition

$$
n_{e}\left(n_{e}+1\right)=-a^{2} \frac{\left\langle\Psi^{\prime} \nabla^{2} \Psi^{\prime}\right\rangle}{\left\langle\Psi^{\prime 2}\right\rangle} .
$$

The squared energy-containing wavenumber $n_{e}\left(n_{e}+1\right)$, then, is a nondimensional ratio of barotropic eddy kinetic energy to streamfunction variance. It is analogous to a squared Rossby wavenumber, which can be interpreted as a nondimensional ratio of eddy available 
potential energy to (baroclinic) streamfunction variance.

For a sufficiently steep spectrum $E_{n}$, the energycontaining wavenumber $n_{e}$ is close to the maximum of the spectrum, and this is indeed the case in Earth's atmosphere and in our simulations (Figs. 1 and D1). The energy-containing wavenumber defined by (D1) is typically closer to the maximum of the spectrum than the conventional integral wavenumber defined as the first moment of the energy spectrum. We prefer the energy-containing wavenumber defined by (D1) to the conventional integral wavenumber because of its proximity to the maximum of the spectrum and because of its analogy to the Rossby wavenumber as the root of a nondimensional ratio of eddy energy to streamfunction variance.

\section{b. Rossby radius}

We computed the Rossby radius (5) as

$$
L_{R}=c_{R} \frac{N_{p}\left(\bar{p}_{s}-\bar{p}_{t}\right)}{f} \min \left(S_{c}, 1\right),
$$

with the empirical constant $c_{R}=1.35$. Since the inner Rossby radius should not exceed the outer Rossby radius, we replaced the supercriticality in the Rossby radius (5) by $\min \left(S_{c}, 1\right)$. This affects only the few simulations in which the supercriticality exceeds one (primarily those with $\gamma=1.0$ and with small surface temperature gradients; see section 3). Surface averages are again interpreted as near-surface averages. Like the energy-containing scale, the Rossby radius is expressed as the spherical wavenumber $n_{R}$ for which $n_{R}\left(n_{R}+1\right)$ $=a^{2} / L_{R}^{2}$.

The empirical constant $c_{R}$ was chosen so that the Rossby wavenumber is close to the energy-containing wavenumber in the simulations with the smallest eddy energies (cf. Fig. 6). The resulting Rossby wavenumber is approximately equal to the wavenumber of maximum baroclinic conversion rate from eddy available potential energy to eddy kinetic energy (with slight deviations in the simulations in which the scale of maximum baroclinic conversion rate and the energy-containing scale appear to be limited by the width of the baroclinic zone).

Considering approximations of eigenvalues of the Sturm-Liouville operator $\partial_{p}\left(f^{2} / N_{p}^{2}\right) \partial_{p}$ for quasigeostrophic vertical modes in pressure coordinates (with $N_{p}$ now taken to vary with pressure, rather than being a surface quantity) leads to other outer Rossby radii. For example, a Wentzel-Kramers-Brillouin-Jeffreys (WKBJ) approximation of the vertical-mode eigenvalue problem with the tropopause as an upper bound-

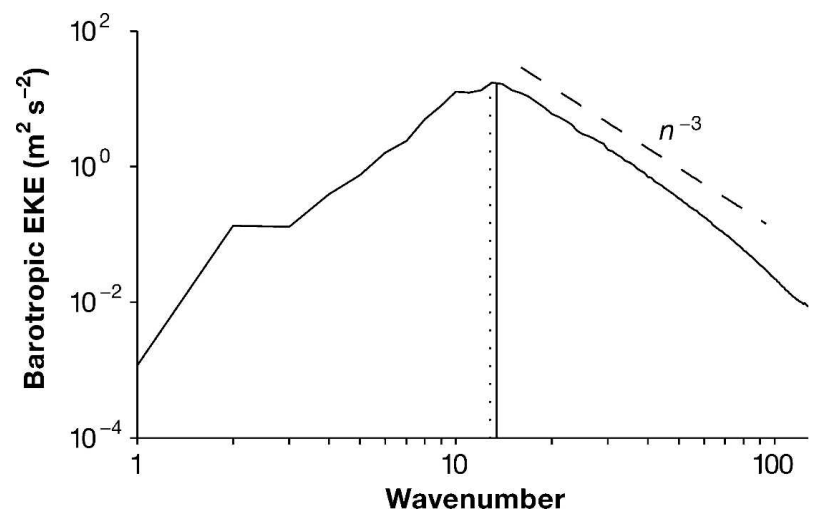

FIG. D1. Wavenumber spectrum of barotropic eddy kinetic energy. Vertical lines indicate characteristic wavenumbers: Rhines wavenumber (dotted), Rossby wavenumber (dash-dotted), and energy-containing wavenumber (solid), listed in order of increasing wavenumber (Rossby wavenumber and energy-containing wavenumber are almost indistinguishable). The dashed line indicates an $n^{-3}$ power law. This barotropic eddy energy spectrum (for a simulation with $\Delta_{h}=240 \mathrm{~K}$ and radius four times that of Earth) is computed in the same way as the spectrum for Earth's atmosphere shown in Fig. 1. It is representative of the spectra seen in the simulations. However, the spectra do not always exhibit an approximate $n^{-3}$ power law but are sometimes steeper.

ary (with any natural boundary condition) leads to an outer Rossby radius proportional to

$$
f^{-1} \int_{\bar{p}_{t}}^{\bar{p}_{s}} N_{p} d p
$$

(see, e.g., Morse and Feshbach 1953, chapter 9.3). Using such an outer Rossby radius does not change the results substantially (except for simulations with $\gamma=$ 1.0 and with small surface temperature gradients; see section 3). For consistency with the scaling arguments presented, we prefer to use the outer Rossby radius $N_{p}\left(\bar{p}_{s}-\bar{p}_{t}\right) / f$ with near-surface averages in the stability measure $N_{p}$.

Figure D1 shows the Rossby wavenumber in relation to the energy spectrum in a typical simulation. The Rossby wavenumber is close to the energy-containing wavenumber. There is no evidence for an inverse energy cascade, such as a flatter $\left(n^{-5 / 3}\right)$ power-law range of the spectrum at wavenumbers smaller than the Rossby wavenumber.

\section{c. Rhines scale}

We computed the Rhines scale (6) as

$$
L_{\beta}=c_{\beta} \frac{\mathrm{EKE}_{\mathrm{bt}}^{1 / 4}}{\beta^{1 / 2}},
$$

with the empirical constant $c_{\beta}=1$. Like the energycontaining scale and the Rossby radius, the Rhines scale is expressed as the spherical wavenumber $n_{\beta}$ for 
which $n_{\beta}\left(n_{\beta}+1\right)=a^{2} / L_{\beta}^{2}$. The empirical constant $c_{\beta}$ was chosen so that the Rhines wavenumber is close to the energy-containing wavenumber in the simulations with the largest eddy energies. The barotropic eddy kinetic energy $\mathrm{EKE}_{\mathrm{bt}}$ was averaged over baroclinic zones, and a reference value was used for $\beta$ (see appendix C); using global averages of barotropic eddy kinetic energy in place of averages over baroclinic zones does not affect the results substantially.

Figure D1 shows the Rhines wavenumber in relation to the energy-containing wavenumber and the Rossby wavenumber. As in the spectrum shown, if $S_{c} \sim 1$, the Rhines wavenumber is close to the energy-containing wavenumber and thus is close to the maximum of the energy spectrum (cf. Fig. 5).

\section{REFERENCES}

Betts, A. K., 1986: A new convective adjustment scheme. Part I: Observational and theoretical basis. Quart. J. Roy. Meteor. Soc., 112, 677-691.

_ , and M. J. Miller, 1986: A new convective adjustment scheme. Part II: Single column tests using GATE wave, BOMEX, ATEX and arctic air-mass data sets. Quart. J. Roy. Meteor. Soc., 112, 693-709.

Boer, G. J., 1983: Homogeneous and isotropic turbulence on the sphere. J. Atmos. Sci., 40, 154-163.

— bulence in the atmosphere. J. Atmos. Sci., 40, 164-184.

Bourke, W., 1974: A multilevel spectral model. I. Formulation and hemispheric integrations. Mon. Wea. Rev., 102, 687-701.

Charney, J. G., 1971: Geostrophic turbulence. J. Atmos. Sci., 28, 1087-1095.

Cho, J. Y.-K., and L. M. Polvani, 1996: The morphogenesis of bands and zonal winds in the atmospheres on the giant outer planets. Science, 273, 335-337.

Frederiksen, J. S., 1983: Disturbances and eddy fluxes in Northern Hemisphere flows: Instability of three-dimensional January and July flows. J. Atmos. Sci., 40, 836-855.

Held, I. M., 1978: The vertical scale of an unstable baroclinic wave and its importance for eddy heat flux parameterizations. $J$. Atmos. Sci., 35, 572-576.

— 1982: On the height of the tropopause and the static stability of the troposphere. J. Atmos. Sci., 39, 412-417.

—_, and M. J. Suarez, 1994: A proposal for the intercomparison of the dynamical cores of atmospheric general circulation models. Bull. Amer. Meteor. Soc., 75, 1825-1830.

- and V. D. Larichev, 1996: A scaling theory for horizontally homogeneous, baroclinically unstable flow on a beta-plane. $J$. Atmos. Sci., 53, 946-952.

— - and T. Schneider, 1999: The surface branch of the zonally averaged mass transport circulation in the troposphere. $J$. Atmos. Sci., 56, 1688-1697.

Holloway, G., 1986: Estimation of oceanic eddy transports from satellite altimetry. Nature, 323, 243-244.

Ingersoll, A. P., and H. Kanamori, 1995: Waves from the collisions of comet Shoemaker-Levy 9 with Jupiter. Nature, 374, 706708.

— , P. J. Gierasch, D. Banfield, A. R. Vasavada, and the Galileo Imaging Team, 2000: Moist convection as an energy source for the large-scale motions in Jupiter's atmosphere. Nature, 403, 630-632.

_ and Coauthors, 2004: Dynamics of Jupiter's atmosphere. Jupiter: The Planet, Satellites, and Magnetosphere, F. Bagenal, T. E. Dowling, and W. B. McKinnon, Eds., Cambridge University Press, $748 \mathrm{pp}$.

Kållberg, P., A. Simmons, S. Uppala, and M. Fuentes, 2004: The ERA-40 archive. Tech. Rep., European Centre for MediumRange Weather Forecasts, Reading, United Kingdom, 31 pp. [Available online at www.ecmwf.int/publications.]

Kushner, P. J., and I. M. Held, 1998: A test, using atmospheric data, of a method for estimating oceanic eddy diffusivity. Geophys. Res. Lett., 25, 4213-4216.

Lapeyre, G., and I. M. Held, 2003: Diffusivity, kinetic energy dissipation, and closure theories for the poleward eddy heat flux. J. Atmos. Sci., 60, 2907-2916.

Lau, N.-G., 1978: On the three-dimensional structure of the observed transient eddy statistics of the Northern Hemisphere wintertime circulation. J. Atmos. Sci., 35, 1900-1923.

Lindzen, R. S., 1993: Baroclinic neutrality and the tropopause. J. Atmos. Sci., 50, 1148-1151.

— , and B. Farrell, 1980a: The role of the polar regions in global climate, and a new parameterization of global heat transport. Mon. Wea. Rev., 108, 2064-2079.

—, and - 1980b: A simple approximate result for the maximum growth rate of baroclinic instabilities. J. Atmos. Sci., 37, $1648-1654$

Lorenz, E. N., 1955: Available potential energy and the maintenance of the general circulation. Tellus, 7, 157-167.

Morse, P. M., and H. Feshbach, 1953: Methods of Theoretical Physics. International Series in Pure and Applied Physics, McGraw-Hill, 1978 pp.

Panetta, R. L., 1993: Zonal jets in wide baroclinically unstable regions: Persistence and scale separation. J. Atmos. Sci., 50, 2073-2106.

Pedlosky, J., 1970: Finite-amplitude baroclinic waves. J. Atmos. Sci., 27, 15-30.

, 1979: Finite-amplitude baroclinic waves in a continuous model of the atmosphere. J. Atmos. Sci., 36, 1908-1924.

Rhines, P. B., 1975: Waves and turbulence on a $\beta$-plane. J. Fluid Mech., 69, 417-443.

Salmon, R., 1980: Baroclinic instability and geostrophic turbulence. Geophys. Astrophys. Fluid Dyn., 15, 167-211.

Santer, B. D., and Coauthors, 2003: Contributions of anthropogenic and natural forcing to recent tropopause height changes. Science, 301, 479-483.

Schneider, T., 2004: The tropopause and the thermal stratification in the extratropics of a dry atmosphere. J. Atmos. Sci., 61, 1317-1340.

_ 2 2005: Zonal momentum balance, potential vorticity dynamics, and mass fluxes on near-surface isentropes. J. Atmos. Sci., 62, 1884-1900.

— 2006: The thermal stratification of the extratropical troposphere. The Global Circulation of the Atmosphere: Phenomena, Theory, Challenges, T. Schneider and A. H. Sobel, Eds., Princeton University Press, in press. [Available online at www.gps.caltech.edu/ tapio.]

Shepherd, T. G., 1987: A spectral view of nonlinear fluxes and stationary-transient interaction in the atmosphere. J. Atmos. Sci., 44, 1166-1178.

Simmons, A. J., and B. J. Hoskins, 1976: Baroclinic instability on the sphere: Normal modes of the primitive and quasigeostrophic equations. J. Atmos. Sci., 33, 1454-1477. 
— , and — 1977: Baroclinic instability on the sphere: Solutions with a more realistic tropopause. J. Atmos. Sci., 34, 581-588.

Smagorinsky, J., S. Manabe, and J. L. Holloway Jr., 1965: Numerical results from a nine-level general circulation model of the atmosphere. Mon. Wea. Rev., 93, 727-768.

Smith, K. S., and G. K. Vallis, 2002: The scales and equilibration of midocean eddies: Forced-dissipative flow. J. Phys. Oceanogr., 32, 1699-1720.

Stone, P. H., 1978: Baroclinic adjustment. J. Atmos. Sci., 35, 561571.

, and B. Nemet, 1996: Baroclinic adjustment: A comparison between theory, observations, and models. J. Atmos. Sci., 53, $1663-1674$.

Straus, D. M., and P. Ditlevsen, 1999: Two-dimensional turbulence properties of the ECMWF reanalyses. Tellus, 51A, 749-772.

Thuburn, J., and G. C. Craig, 1997: GCM tests of theories for the height of the tropopause. J. Atmos. Sci., 54, 869-882.

Valdes, P. J., and B. J. Hoskins, 1988: Baroclinic instability of the zonally averaged flow with boundary layer damping. $J$. Atmos. Sci., 45, 1584-1593.

Vallis, G. K., and M. E. Maltrud, 1993: Generation of mean flows and jets on a beta plane and over topography. J. Phys. Oceanogr., 23, 1346-1362. 